\title{
Improving the capability of an integrated CA-Markov model to simulate spatio- temporal urban growth trends using an Analytical Hierarchy Process and Frequency Ratio
}

\begin{abstract}
The creation of an accurate simulation of future urban growth is considered one of the most important challenges in urban studies that involve spatial modeling. The purpose of this study is to improve the simulation capability of an integrated CA-Markov Chain (CA-MC) model using CA-MC based on the Analytical Hierarchy Process (AHP) and CA-MC based on Frequency Ratio (FR), both applied in Seremban, Malaysia, as well as to compare the performance and accuracy between the traditional and hybrid models. Various physical, socio-economic, utilities, and environmental criteria were used as predictors, including elevation, slope, soil texture, population density, distance to commercial area, distance to educational area, distance to residential area, distance to industrial area, distance to roads, distance to highway, distance to railway, distance to power line, distance to stream, and land cover. For calibration, three models were applied to simulate urban growth trends in 2010; the actual data of 2010 were used for model validation utilizing the Relative Operating Characteristic (ROC) and Kappa coefficient methods Consequently, future urban growth maps of 2020 and 2030 were created. The validation findings confirm that the integration of the CA-MC model with the FR model and employing the significant driving force of urban growth in the simulation process have resulted in the improved simulation capability of the CA-MC model. This study has provided a novel approach for improving the CA-MC model based on FR, which will provide powerful support to planners and decision-makers in the development of future sustainable urban planning.
\end{abstract}

Keyword: CA-Markov; Analytical hierarchy process; Frequency ratio; Urban growth; Simulation 